\title{
Multi-stock replenishment of naval vessels by specialist replenishment ships
}

\author{
S.J. Rowling ${ }^{\text {(iD) }}$, M. Teo ${ }^{\text {a }(D)}$ and $\underline{\text { M.B.A. West }}{ }^{\text {a }}$ \\ a Joint and Operations Analysis Division, Defence Science and Technology Group, Sydney, Australia \\ Email: marc.west@dst.defence.gov.au
}

\begin{abstract}
Naval replenishment vessels play an important role in military operations as they resupply and sustain other military units, allowing them to react quickly and perform their required roles for an extended period of time. They may replenish naval task groups with fuel (both maritime fuel and aviation fuel for helicopters), stocks (food, ammunition etc.), and also may support deployed land forces.

This study examines the trade-offs between various replenishment ship types supporting naval operations, and finds through sensitivity analysis that the level of risk regarding fuel and stock availability a task group of ships wishes to take on is a key factor in determining an optimal replenishment fleet. A scheduling model was built in which a task group of military assets was replenished indefinitely by replenishment vessels, which travelled back and forth to a distant port to restock themselves. Subject to constraints and demands, a number of measures of effectiveness were developed in order to examine the replenishment ship fleet. These include the amount of time a replenishment ship is not needed and remains in port (slack time) within a particular schedule, and the maximum distance off shore a combination of replenishment ships can support naval and joint operations.
\end{abstract}

Keywords: Operations Analysis, scheduling, replenishment, optimisation 


\section{INTRODUCTION}

The sizing of a Naval fleet, and the scheduling of replenishment at sea, has been the subject of numerous studies; for example (Williams et al. 1989, Pilnik et al. 1991, Dunn 1992, Chircop et al. 2013, Brown et al. 2018). The Defence White Paper 2016 states that the Royal Australian Navy (RAN) Supply-class oilers will be supplemented by a "third replenishment or additional logistics vessel" in the late 2020s (Commonwealth of Australia 2016). The RAN is also set to upgrade its HMAS Choules-style "logistics" ship, so the decision for the RAN as stated in the White Paper is to procure either a third oiler, primarily designed for fuel resupply with limited stores capacity, or a second logistics ship, which has little refuelling capability but a greater stores capacity. In addition to these two options, the RAN is now also considering the possibility of procuring two "hybrid" ships, rather than upgrading the logistics ship. Hybrid ships have a range of storage and replenishment capacities.

In response to the Defence White Paper statement, a bespoke model was developed that met RAN requirements pertaining to operational scenarios, fuel and stock consumption and storage, and ship types. Naval operations have many constraints; for example, vessels must maintain a minimum fuel level. Ships consume fuel and stores at various rates, depending on what operations they are conducting, and also have differing storage capacities; for example, a Landing Helicopter Dock (LHD) can carry far more of most stocks than the surface combatants. The ships may also be supporting a landing force, which has its own consumption and storage parameters, or a fleet of helicopters, which consume a different type of fuel to the ships.

In the first phase of model development, the RAN's requirement for an additional replenishment ship to carry maritime fuel was studied by considering the refuelling of a three surface combatant (SC) task group. The model was then expanded to consider the resupply of stores, as well as fuel. The stocks considered are: Maritime fuel (F76), Aviation fuel (F44), Explosive Ordnance (EO), Food and Other. Fresh water has not been considered, as ships can generate water themselves. This expanded model considers the refuelling and restocking of a larger task force (two LHDs, one Landing Ship Dock (LSD) and seven SCs) and a land force. This larger task group, particularly the LHD and LSD, is needed to support the land force. We model a steady state replenishment operation, and do not consider the deployment and return of the task group. Ship maintenance and crew scheduling is also not considered. A replenishment ship carrying fuel will carry bunker fuel, used by the ship for propulsion and standby power requirements, and cargo fuel, used to refuel other ships. We have assumed that fuel can be transferred between the bunker and cargo when needed. If this assumption is not made, there are serious limitations on how far an oiler can travel from a refuelling port.

\section{MODELLING}

\subsection{Refuelling}

As part of initial model development, the refuelling of a small task group by a single class of replenishment ship was considered. Auxiliary Oiler Replenishment ships ("Oilers" or AORs) travel to a site, remain there for the duration of a single refuel of the task group and return to port to refuel. This process can be repeated for any number of sites as long as the oiler is able to return to the site before the task group runs out of fuel. It is assumed that the task group is ready to be refuelled immediately when the oiler arrives. Representative data are used throughout this article to highlight the important facets of the replenishment operation that must be taken into account throughout the acquisition process of new ships. Data and sources can be provided on request. The time an oiler spends on site to refuel a task group and the time it spends refuelling at port is calculated based on the amount of fuel being transferred, fuel consumption rates and the fuel flow rate. It is also assumed that all ships maintain a $30 \%$ fuel reserve; this number was chosen as a representative fuel reserve as it is typical of Navy vessels (Baggett 2008). The methodology for multi-sites is included below, however only results for a single site are shown. 


\section{Single site refuelling}

Figure 1 depicts the operation of one and two oilers in servicing the task group. This method is called Shuttling. Recall that the oiler needs to return back to the task group before the task group runs out of fuel. Let

- $n$ be the number of oilers,

- $T_{T G}$ be the time between refuels of the task group,

- $\quad T_{A P}=T_{P A}$ be the travel time of an oiler between the site $(\mathrm{A})$ and port $(\mathrm{P})$,

- $T_{R T G}$ be the time taken for an oiler to refuel the task group at the site,

- $T_{R}$ be the time taken to refuel an oiler at port, and

- $T_{S}$ be the available slack time.

Then, in order for the task group to be serviced by the oilers, the following inequality,

$T_{R}+2 T_{A P}+T_{R T G}+T_{S} \leq n T_{T G}$,

must be satisfied. There are two measures of effectiveness (MOEs): the available slack time $T_{S}$ (the amount of time the oilers are not performing their duties) and the maximum deployment range of the task group for varying number of oilers, assuming zero slack time.

\section{Multi-site refuelling}

Two methods of refuelling are considered: Shuttling and Racetrack. The racetrack method is similar to the shuttling method in that the amount of time an oiler remains on site is the duration of a single refuel of the task group. The main difference between the two modes is that for the racetrack method, the oiler visits all sites before returning to port to refuel. These methods can be generalised to many sites, however the RAN would only rarely conduct more than two naval operations requiring refuelling simultaneously. Hence, we detail the approach for two concurrent operations. Figure 2 depicts the operation of a single oiler servicing two sites sequentially using the shuttling and racetrack methods. The use of more than one oiler to service the sites is similar, with the exception of how often each oiler visits the sites. Let

- $\quad n$ be the number of oilers,

- $\quad T_{T G A}$ and $T_{T G B}$ be the time between refuels of the task groups at site $A$ and site $B$ respectively,

- $\quad T_{T G}=\min \left(T_{T G A}, T_{T G B}\right)$ be the time between refuels of both task groups,

- $\quad T_{A P}=T_{P A}$ be the travel time between site $\mathrm{A}$ and port $(\mathrm{P})$,

- $\quad T_{B P}=T_{P B}$ be the travel time between site $\mathrm{B}$ and port $(\mathrm{P})$,

- $T_{A B}=T_{B A}$ be the travel time between site $\mathrm{A}$ and site $\mathrm{B}$,

- $T_{R A}$ and $T_{R B}$ be the time taken to refuel the oiler at port after visiting site A and site $\mathrm{B}$ respectively,

- $T_{R}$ be the time taken to refuel the oiler at port after visiting both sites,

- $T_{R T G A}$ and $T_{R T G B}$ be the time taken to refuel the task groups at site A and site B respectively,

- $\quad T_{S A}$ and $T_{S B}$ be the slack time after visiting site $\mathrm{A}$ and site B respectively, and

- $T_{S}$ be the total slack time.

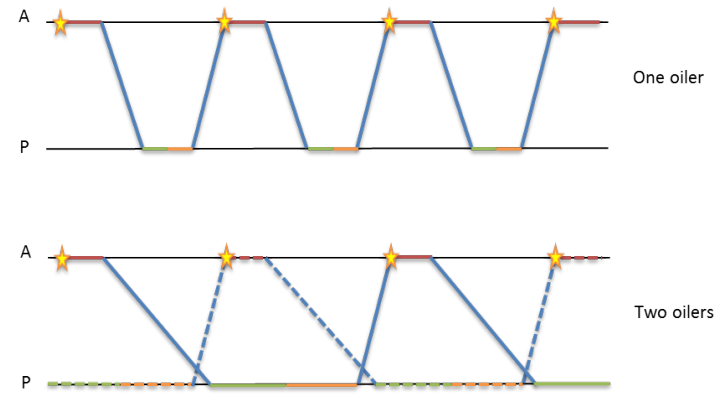

Figure 1. One (top) and two (bottom) oilers servicing a single site. The stars indicate when the task group needs refuelling, the red lines represent blue lines represent the transit of an oiler site $(\mathrm{A})$ and port $(\mathrm{P})$, the green lines ent the orange lines represent the available slack time. For the case of two oilers, the solid and dashed lines depict the operation of each oiler.

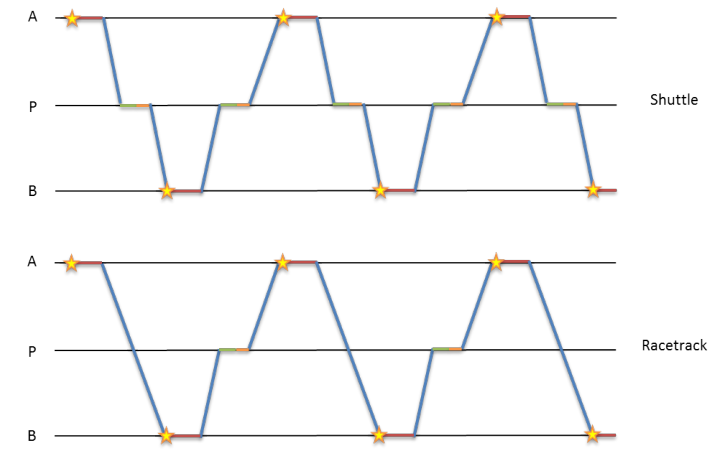

Figure 2. Example of the shuttling (top) and racetrack (bottom) methods for a single oiler servicing two sites concurrently. The stars indicate when the task group needs refuelling, the red lines represent the time the oiler spends refuelling the task group, the blue lines represent the transit of an oiler between the sites (A and B) and port (P), the green lines represent the refuelling of the oiler at port and the orange lines represent the available slack time. the time an oiler spends refuelling the task group, 
Note that the time between refuels of both task groups, $T_{T G}$, is calculated by taking the minimum of the time between refuels of each task group to ensure that the oiler returns to the task groups before either task group runs out of fuel. In order for both task groups to be serviced by $n$ oilers using the shuttling method, the following inequality must be satisfied,

$T_{R T G A}+T_{R T G B}+T_{R A}+T_{R B}+2 T_{A P}+2 T_{B P}+T_{S A}+T_{S B} \leq n T_{T G}$,

Similarly, the following inequality,

$T_{R T G A}+T_{R T G B}+T_{R}+T_{A B}+T_{B P}+T_{A P}+T_{S} \leq n T_{T G}$,

must be satisfied for both task groups to be serviced by $n$ oilers using the racetrack method. As with the case for a single site, we can obtain the two MOEs of interest, slack time and maximum deployment range for varying number of oilers assuming zero slack time, by rearranging (2) and (3).

\subsection{Restocking and Refuelling}

The refuelling model was expanded to consider the resupply of various stocks, as well as fuel, to a large task group supporting land forces. Replenishment ships operate on a continuous shuttle between theatre and the resupply port, and a periodic shipping schedule is sought that may be repeated as long as ships remain available. The stocks are considered in a similar fashion to fuel in the various inequalities, with storage capacities and consumption/restock rates contributing to the various time components. The schedule begins with the arrival in theatre of the first replenishment ship. Subsequent deliveries are scheduled to arrive "just in time" based on inventory levels and consumption rates. The amount of stock delivered in each shipment is limited by either the ship's capacity or the

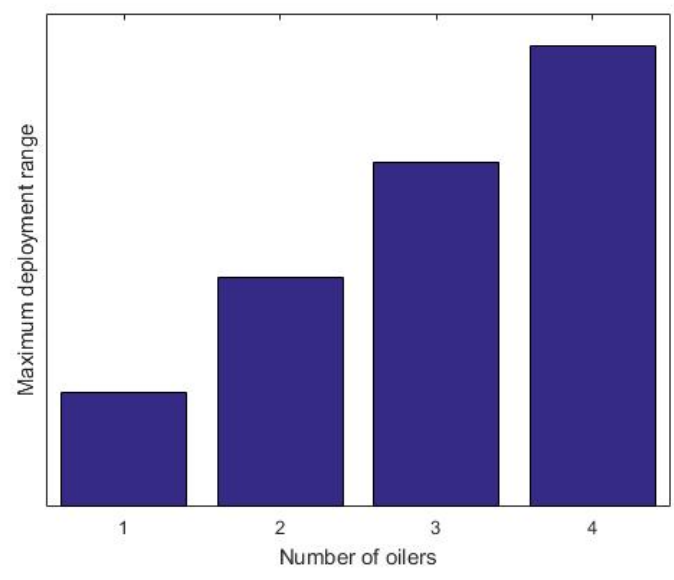

Figure 3. Maximum deployment range of a three surface combatant task group for varying number of oilers.

ability to store goods in theatre. Following the scheduled delivery by the last replenishment ship, we calculate if the resultant inventory will sustain the deployed forces until the return of the first replenishment vessel. If so, inventory may be maintained as long as the replenishment ships are available. In heterogeneous replenishment fleets, the order of arrival may be important. Since the possible fleets for the present problem are small, all possible permutations may be calculated. Replenishment ships are allotted time in port for loading of cargo and turn-around maintenance, as well as time to deliver cargo in theatre. However, no slack time has been allowed in the schedule. The LHD can store goods in theatre and it is assumed there is no landbased storage capacity. Shipping is based on a repeatable, periodic schedule. The overall pace of the schedule is dictated by the slowest ship in the replenishment fleet, which maintains appropriate spacing between ship arrivals. This enables the achievement of stable inventory levels and prevents ships arriving simultaneously.

Ships have been modelled with various storage capacities and consumption rates for the stocks and fuel (F76, F44, EO, Food, Other). Surface combatants (SCs), Supply-class oilers (AORs), Bay-class logistics ships (LSDs), Landing Helicopter Docks (LHDs), and three exemplar "hybrid" ships have been included in the modelling. These hybrids have been designated R1, R2 and R3. The exemplar ships have different storage capacities and are used to draw out the important facets of the replenishment operation. For example, R3 is a larger ship with greater fuel and EO storage capacity; however it is slower and burns fuel at a quicker rate. R1 and R2 are similar ships, with R2 having a slightly larger fuel and EO capacity and smaller with regards the other items. All three travel more quickly than the AORs. Resupply can be conducted by mixes of the AORs, LSDs and hybrids. We have displayed results parametrically to focus on the style of results that the model can produce and the important aspects of the problem, rather than one particular ship combination. Validation and verification of the model was undertaken through consultation with Navy and Defence clients, comparing the results to existing models and by two independent researchers implementing the algorithms separately using different methods. These details can be provided on request. 


\section{SINGLE SITE RESULTS}

\subsection{Refuelling}

For the three surface combatant task group, Figure 3 depicts the maximum deployment range of the task group for varying number of oilers. As one would expect, more oilers result in longer deployment ranges, and the relationship between the number of oilers and the maximum deployment range is a step function. Figure 4 presents the proportion of time the oiler is not in use (slack time) and performing its required tasks (travelling, refuelling the task group, refuelling at port). This allows us to see if the oiler(s) is constantly utilised or if there is any spare capacity. We observe that, for a given range $d$, an increase in the number of oilers results in an increase in the proportion of available slack time whilst, for a given number of

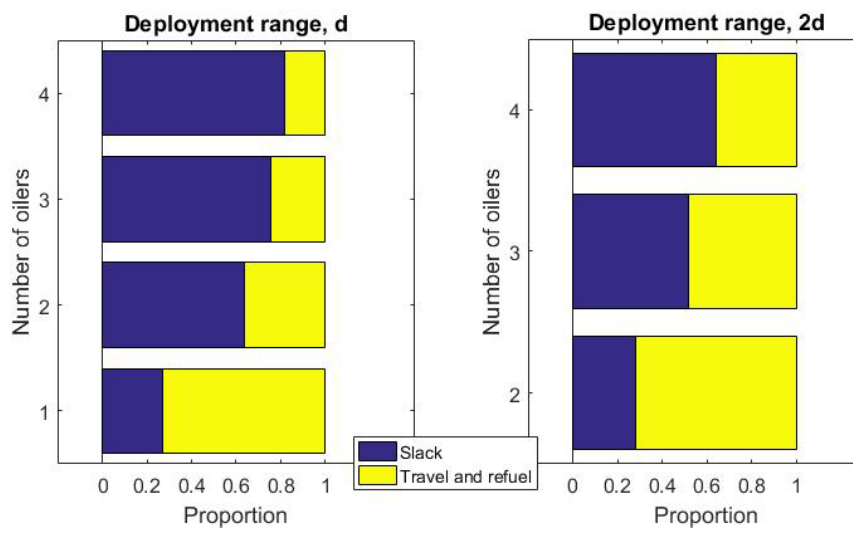

Figure 4. Proportion of time the oiler(s) is not in use (slack time) and operational. The proportions shown are for each oiler operating in tandem with other oilers.

oilers, an increase in the range $(2 d)$ decreases the proportion of available slack time.

\subsection{Restocking and refuelling}

\section{Maximum Deployment Range}

For the large seven surface combatant + LHD + LSD task group, Figure 5 compares the maximum deployment range for a number of replenishment fleet options. This distance depends on the number of replenishment ships in the fleet, with the distances for all the two-ship fleets being less than the three-ship fleets. The one exception is the 2AOR fleet, which has a slightly longer maximum deployment range than the AOR + 2R3 combination. The two-ship fleet options that contain at least one dedicated replenishment ship (AOR, R1, R2, R3) have longer ranges than those that contain the LSD or LHD. This indicates that F76 capacity is a significant factor.
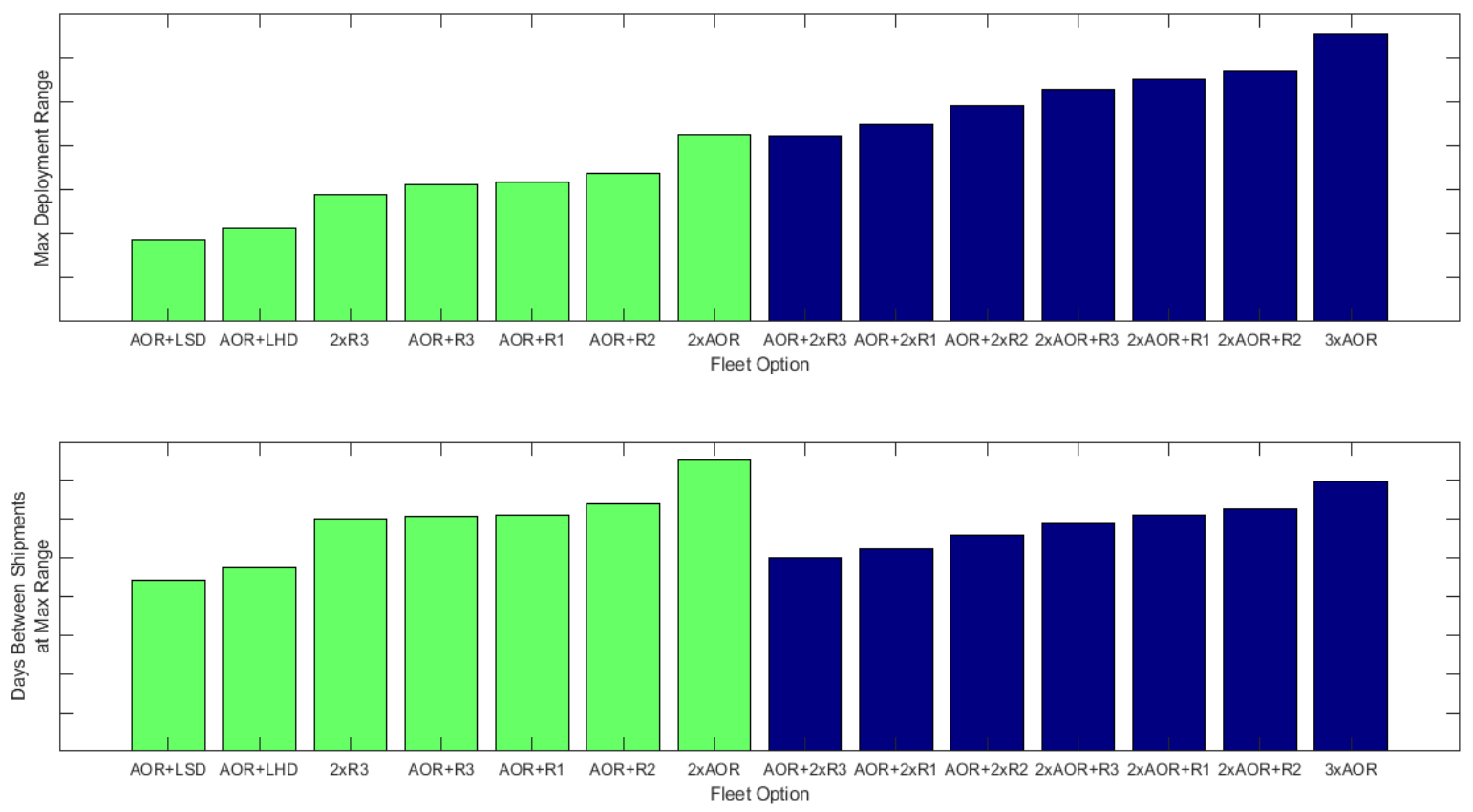

Figure 5. Above: Maximum range at which two-ship (green) and three-ship (blue) fleet options are able to supply goods at the required rate. Below: Time between replenishment shipments when operating at the maximum range for that fleet option. 
Figure 6, Figure 7 and Figure 8 show how the maximum deployment ranges decrease as the consumption rates increase. Figure 6 shows that as the F76 consumption rate is increased, the maximum deployment range decreases for all replenishment fleet options; F76 is a critical driver of the replenishment schedule. Hence, if a replenishment fleet can only just service a particular distance, then any increase in F76 demand will mean that the operational requirements cannot be met.

Conversely, Figure 7 shows that all fleet options are able to absorb a substantial increase in EO consumption with minimal impact to the maximum deployment range. The R3 has the largest EO store of the modelled vessels and so fleets incorporating an R3 are even less sensitive to increased EO demand.

Figure 8 shows the effect of increased demand for F44, which would represent a surge in helicopter activity or the landing forces exceeding their daily ration. Again, the replenishment ships can absorb an increased demand for F44, although not to the same extent as EO.

\section{Single-Unit Endurance Limitations}

Thus far, we have considered the bulk storage capacities and consumption rates of goods in theatre. However, individual units have their own storage restrictions and consumption rates. If goods are able to be redistributed in theatre, then only aggregate consumption needs to be considered. For most goods, we assume that redistribution is possible using helicopters, vehicles and small craft. The exception is fuel. The LHDs can store large volumes of fuel but cannot easily transfer it.

At some point, an individual SC may exhaust its F76 or F44 stock and so will need to be refuelled. If SCs exhaust their fuel supplies quicker than the resupply period indicated in Figure 5, then the replenishment fleet becomes limited by supply frequency rather than storage capacity. This is particularly pertinent for a fleet of 2 AORs. With their high fuel capacity, the AORs offer the greatest deployment range for any 2ship fleet option. However, this extended range, combined with a slower transit speed, results in less frequent deliveries. As the predicted endurance of SCs approaches this delivery frequency, there is a mounting risk to the fuel supply of SCs and helicopters; the system cannot tolerate delays in the replenishment shuttle, or any temporary elevation in F76 or F44 fuel consumption.

Reliance on a two-ship scheme constitutes a risk, which may be mitigated by either:

1. adding a third ship to the replenishment fleet to increase frequency of delivery;

2. locating a refuelling capability in theatre by:

a. attaching an additional replenishment ship to the task group;

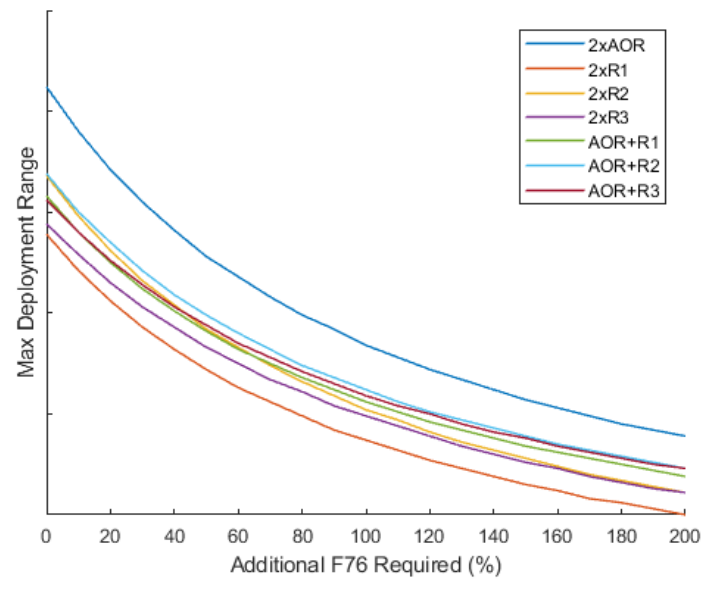

Figure 6. Maximum range at which given fleet options are able to supply the required quantity of goods as the F76 demand is increased. (All other consumption rates held constant.)

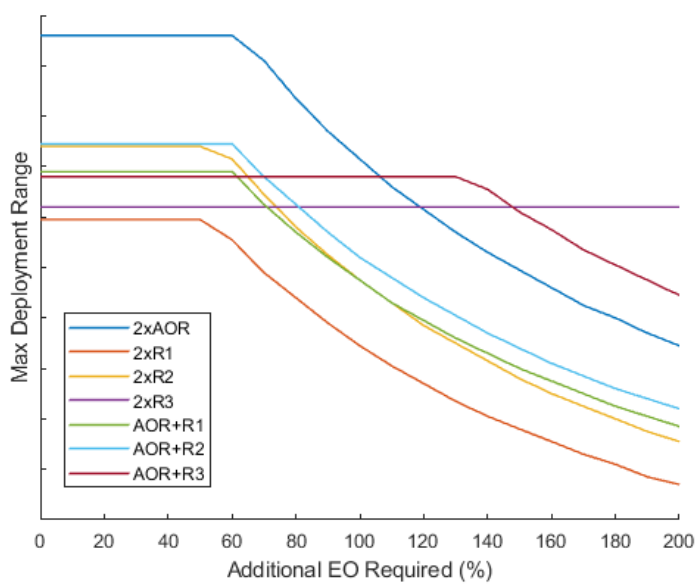

Figure 7. Maximum range at which given fleet options are able to supply the required quantity of goods as the EO demand is increased. (All other consumption rates held constant.)

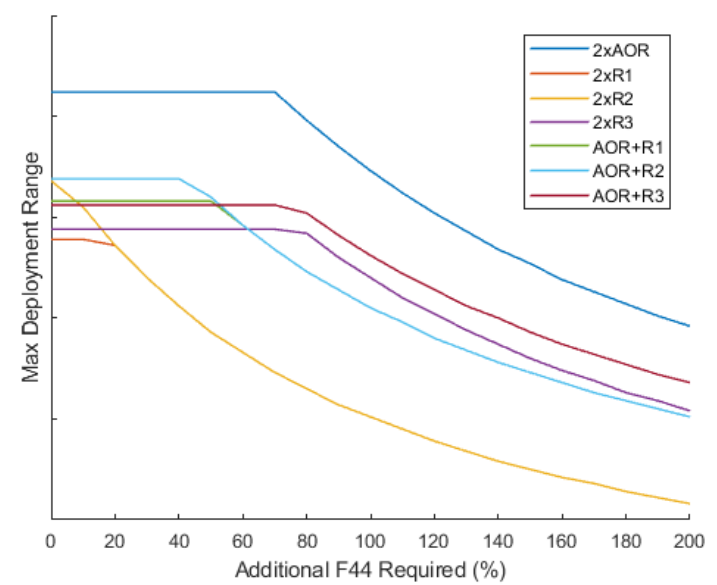

Figure 8. Maximum range at which given fleet options are able to supply the required quantity of goods as the F44 demand is increased. (All other consumption rates held constant.) 
b. fitting a refuelling capability to the LHDs;

c. replacing the LSD in the task group with a R3 that can carry more F44.

Options (1) and (2a) are simply different employment options for a fleet of three dedicated replenishment ships. Options (2b) and (2c) represent two concepts for combining support capabilities in a single vessel.

\section{CONCLUSIONS}

The choice of replenishment ships is not only a matter of supply and consumption, but also a question of risk. Depending on the required operational distance, a third replenishment ship may be a risk-mitigation strategy rather than a requirement, as the two-ship shuttle may deliver the required goods and fuel, but with no refuelling slack time. Sensitivity analysis highlights the importance of either a three-ship replenishment shuttle, or basing one with the task group. An additional benefit to an embedded refuelling capability is that it creates a greater local reserve of fuel, which allows for a high readiness level if fuel-thirsty operations need to be undertaken; for example, manoeuvre to counter a high speed threat or piracy (Mirshak et al. 2010, West et al. 2010, West 2014). The downside is that the task group must provide protection to the high value refuelling asset (West 2003). Sensitivity analysis highlights the utility of the model to quickly examine the vulnerability of the replenishment schedule to increases in fuel and store requirements without time-consuming simulation.

\section{ACKNOWLEDGEMENTS}

The authors would like to acknowledge Andrew Donohoo, Anne Quill and Bernard Kachoyan, upon whose work this study built, Paul Chircop for his learned reviews, and the MODSIM peer reviewers.

\section{REFERENCES}

Baggett, J. P. (2008). Logisitcal Analysis of the Littoral Combat Ship (LCS) operating independently in the Pacific, Naval Postgraduate School, Monterey. http://hdl.handle.net/10945/4284

Brown, G. G., DeGrange, W. C., Price, W. L. and Rowe, A. A. (2018). "Scheduling combat logistics force replenishments at sea for the US Navy" Naval Research Logisitics 64(8): 677-693. doi: $10.1002 /$ nav. 21780

Chircop, P., Surendonk, T. J., Van Den Briel, M. and Walsh, T. (2013). A column generation approach for the scheduling of patrol boats to provide complete patrol coverage, 20th International Congress on Modelling and Simulation. Adelaide, Australia: 1110-1116. https://www.mssanz.org.au/modsim2013/D3/chircop.pdf

Commonwealth of Australia (2016). 2016 Defence White Paper. http://www.defence.gov.au/WhitePaper/Docs/2016-Defence-White-Paper.pdf

Dunn, J. S. (1992). Scheduling underway replenishment as a generalised orienteering problem, Naval Postgraduate School, Monterey. http://hdl.handle.net/10945/23768

Mirshak, R., West, M. and Chircop, P. (2010). Risk models for maritime security, area and port force protection, Waterside Security Conference (WSS), 2010 International. Carrara, Italy: 1-8. doi: $10.1109 /$ WSSC.2010.5730263

Pilnik, S. E., Glazebrook, K. D. and Gaver, D. P. (1991). "Optimal Sequential Replenishment of Ships During Combat" Naval Research Logisitics 38: 637-668. doi: 10.1002/1520-6750(199110)38:5\%3C637::AID-NAV3220380502\%3E3.0.CO;2-B

West, M. (2003). High Value Unit Protection during Amphibious Operations, Land Warfare Conference. Adelaide, Australia. doi: 10.13140/RG.2.1.5090.7286

West, M. (2014). "Keep-out ranges for defence of a turning frigate against small boat attack" Australian Defence Operations Research Review 2014. doi: 10.13140/RG.2.2.27444.09600

West, M., Cooper, T. and Kachoyan, B. (2010). "AIS Analysis in Support of Counter-Piracy Operations" Australian Journal of Maritime and Ocean Affairs 2(4). doi: 10.1080/18366503.2010.10815665

Williams, T. M., Gittins, R. P. and Byrke, D. M. (1989). "Replenishment at Sea" Journal of the Operational $\underline{\text { Research Society 40(10): 881-887. doi: } 10.1057 / \text { jors. } 1989.156}$ 